\title{
Large eddy simulations of transitional round jets: Influence of the Reynolds number on flow development and energy dissipation
}

\author{
Christophe Bogey and Christophe Bailly \\ Laboratoire de Mécanique des Fluides et d'Acoustique, UMR CNRS 5509, Ecole Centrale de Lyon, \\ 69134 Ecully, France
}

(Received 20 December 2005; accepted 4 April 2006; published online 6 June 2006)

\begin{abstract}
Transitional round jets at Mach number $M=0.9$, with identical initial conditions except for the diameter, yielding Reynolds numbers over the range $1.7 \times 10^{3} \leqslant \operatorname{Re}_{D} \leqslant 4 \times 10^{5}$, are computed by large eddy simulation (LES) using explicit selective/high-order filtering. The effects of the Reynolds number on the jet flows are first presented. As the Reynolds number decreases, the jets develop more slowly upstream from the end of the potential core, but more rapidly downstream. At lower Reynolds numbers, the decay of the centerline velocity and the jet spreading are indeed faster, and the turbulence intensities are higher after the potential core, in agreement with data of the literature. The integral length scales are also significantly larger. The results suggest moreover that the jet self-similar region is reached at shorter axial distances at lower Reynolds numbers. The influence of the Reynolds number on the energy-dissipation mechanisms involved in the LES, namely molecular viscosity and explicit filtering, is secondly investigated. At high Reynolds number, energy dissipation is mainly ensured by the explicit filtering, through the smaller scales discretized. As the Reynolds number decreases, the contribution of molecular viscosity increases and becomes predominant. Molecular viscosity is also shown to affect a large range of turbulent scales with a dissipation peak observed around the Taylor length scale. (C) 2006 American Institute of Physics.

[DOI: $10.1063 / 1.2204060]$
\end{abstract}

\section{INTRODUCTION}

Successive studies over the years have shown that the development of round free jets depends on the flow initial parameters. ${ }^{1}$ Among these parameters, the influence of the Mach number has been investigated experimentally by $\mathrm{Lau}^{2}$ and Zaman, ${ }^{3}$ who provided measurements of mean flow and turbulence characteristics, and spreading and velocity decay rates for compressible jets over a wide range of Mach numbers. In the same way, Malmström et al. ${ }^{4}$ examined the diffusion of axisymmetric jets for low outlet velocities.

One important parameter in the development of jet flows was found to be the properties of the shear layer at the nozzle exit, such as the profile of mean velocity and the turbulence intensity. Their effects have been documented experimentally by different authors for circular jets at fixed Mach and Reynolds numbers. Ferdman et al. ${ }^{5}$ reported, for instance, the influence of nonuniform initial velocity profiles on the downstream evolution of round jets at the Reynolds number of $2.4 \times 10^{4}$. Raman et al. $^{6}$ and Antonia and co-workers ${ }^{7,8}$ considered jets at Reynolds numbers of $4 \times 10^{5}$ and $8.6 \times 10^{4}$, respectively, displaying either transitional or turbulent nozzle-exit boundary layers. Both concluded that jets with initially transitional shear layer develop more rapidly, with shorter core length and higher velocity decay rate, than jets with initially turbulent shear layer. $\mathrm{Xu}$ and Antonia $^{8}$ moreover noticed that jets approach self-similarity more rapidly when the initial shear layer is laminar. Finally the state of the nozzle-exit boundary layer was shown also to affect significantly the sound field radiated by subsonic jets, in particular by $\operatorname{Zaman}^{9}$ who observed experimentally that initially laminar jets emit more noise.

The dependence of jet noise on the Reynolds number is also significant, and was displayed notably by the experiments of McLaughlin et al. ${ }^{10,11}$ In round jets, the diameterbased Reynolds number $\operatorname{Re}_{D}=u_{j} D / \nu$, where $u_{j}$ and $D$ are the jet exit velocity and diameter, and $\nu$ is the kinematic viscosity, is indeed a key parameter as it indicates the magnitude of the effects of molecular viscosity on the turbulent scales. Analytical works ${ }^{12}$ have for instance shown that the growth of instability waves in the jet shear layers can be appreciably reduced as the Reynolds number decreases. Experiments have also been conducted to investigate the effects of the Reynolds number on jet flows at low Reynolds numbers. Lemieux and Oosthuizen ${ }^{13}$ observed that the characteristics of a plane jet change significantly in the Reynolds number range of 700-4200. Namer and Ötügen ${ }^{14}$ found that lower Reynolds numbers lead to higher jet dilution and spread rates for plane jets with Reynolds numbers between 1000 and 7000. Similar results were obtained recently by Kwon and $\mathrm{Seo}^{15}$ for round jets over the Reynolds number range 4375142. Significant effects on the Reynolds number have also been noted on the instantaneous flow structure, see for example the snapshots provided by Weisgraber and Liepmann ${ }^{16}$ for round jets at Reynolds numbers 5500 and 16000 .

The influence of the Reynolds number on the development of jet flows is however still a discussion topic. This may be partly due to the fact that the initial properties of the shear layer depend strongly on the Reynolds number. The variations of the turbulence intensity and of the boundarylayer thickness at the nozzle exit as a function of the Rey- 
nolds number were for instance documented for round jets by Zaman, ${ }^{17}$ who observed that jets are naturally initially laminar for about $\operatorname{Re}_{D}<10^{5}$, but fully turbulent for about $\operatorname{Re}_{D}>5 \times 10^{5}$. The influence of the Reynolds number on jet features is therefore frequently linked to the effects of the initial shear-layer characteristics, which can be significant as it was mentioned previously. The discrepancies between well-known experimental data ${ }^{11,18-22}$ obtained for round jets at Reynolds numbers over the wide range $3.6 \times 10^{3}<\mathrm{Re}_{D}$ $<10^{6}$ are consequently expected to result both from direct effects of the Reynolds number on the turbulence development and from indirect effects due to alterations in the initial conditions of the shear layer.

Considering these issues, it seems worthwhile to perform numerical simulations to investigate the influence of the Reynolds number in turbulent flows, because in simulations the Reynolds number of the flows can be changed without modifying other inflow parameters. One must yet pay attention to deal with physical configurations. For instance, in jets, transitional initial conditions are not realistic for Reynolds numbers $\operatorname{Re}_{D}>5 \times 10^{5}$. Previously, both direct numerical simulations ${ }^{23,24}$ (DNS) and large eddy simulations ${ }^{25}$ (LES) have been carried out to study the effects of the inflow conditions and forcing on flow and noise for transitional round jets. DNS was also used by Klein et $a l .{ }^{26}$ to investigate the influence of the Reynolds number on a plane jet for Reynolds numbers lower than 6000. Due to computing limitations, DNS is however restricted to low Reynolds numbers, whereas LES, where only the turbulent scales larger than the grid size are calculated, can be theoretically applied to all Reynolds numbers. For the study of Reynolds-number effects, LES therefore appears more appropriate, but it is crucial, in order to obtain accurate results, that the LES methodology does not artificially decrease the effective Reynolds number of the computed flows. That might indeed be the case when modeling based on eddy viscosity is used to take into account the subgrid scales. ${ }^{27-29}$

In the present article, results of LES of transitional round free jets at Mach number $\mathrm{M}=u_{j} / c_{0}=0.9$ (where $c_{0}$ is the speed of sound in the ambient medium) are reported. The simulations have been performed with low-dissipation numerical schemes, ${ }^{30}$ using explicit selective/high-order filtering in order to preserve the Reynolds number $\operatorname{Re}_{D}=u_{j} D / \nu$ set by the inflow parameters. ${ }^{29}$ The initial conditions of the jet flows (shear-layer thickness, inflow forcing) are identical except for the jet diameter $D$, yielding Reynolds numbers $\operatorname{Re}_{D}$ from $1.7 \times 10^{3}$ up to $4 \times 10^{5}$. The main objective is thus to show the influence of the Reynolds number on the jet development in the absence of other initial-condition effects. Our attention will be particularly focused on the transitional region of the jet flow located just after the end of the potential core. LES results will be in particular compared to corresponding data of the literature obtained for Mach 0.9 transitional jets at the low ${ }^{11,31}$ and high ${ }^{22}$ Reynolds numbers $\operatorname{Re}_{D}=3.6 \times 10^{3}$ and $5 \times 10^{5}$ whenever possible. It can also be noted that in previous works the flow and sound fields of the simulated jet at $\operatorname{Re}_{D}=4 \times 10^{5}$ were successfully compared to measurements at high Reynolds numbers, ${ }^{32}$ and that the properties of the noise radiated by the different jets were also presented in detail. ${ }^{33}$ Moreover, in order to study the influence of the Reynolds number on the dissipation mechanisms involved in the simulations, namely molecular viscosity and explicit selective filtering, the budgets of the turbulent kinetic energy are calculated in the jets. In this way, the damping rates and the features in the wave number space for the two dissipation mechanisms will be shown and discussed.

The present article is organized as follows. The numerical procedure and the parameters of the different simulations are first provided. Jet flow features obtained from the LES, including vorticity snapshots, mean flow characteristics, and turbulence intensities, as well as velocity spectra and integral length scales, are then presented. The kinetic energy budgets are subsequently calculated, and the contributions of molecular viscosity and explicit filtering to the energy dissipation are shown. Finally concluding remarks are drawn.

\section{SIMULATION PARAMETERS}

\section{A. Governing equations}

Following the works of Vreman et al., ${ }^{34}$ the filtered compressible Navier-Stokes equations are recasted in the following way:

$$
\begin{aligned}
& \partial_{t} \bar{\rho}+\partial_{j}\left(\bar{\rho} \tilde{u}_{j}\right)=0, \\
& \partial_{t}\left(\bar{\rho} \tilde{u}_{i}\right)+\partial_{j}\left(\bar{\rho} \tilde{u}_{i} \tilde{u}_{j}+\bar{p} \delta_{i j}-\widetilde{\tau}_{i j}\right)=\sigma_{i}^{\mathrm{sgs}}, \\
& \partial_{t}\left(\bar{\rho} \breve{e}_{t}\right)+\partial_{j}\left(\left(\bar{\rho} \breve{e}_{t}+\bar{p}\right) \tilde{u}_{j}+\tilde{q}_{j}-\tilde{\tau}_{i j} \tilde{u}_{i}\right)=\sigma_{e}^{\mathrm{sgs}},
\end{aligned}
$$

where $\rho$ represents the density, $u_{i}$ the velocity, $p$ the pressure, $\tau_{i j}$ the viscous tensor, and $q_{j}$ the heat flux. The overbar denotes a filtered quantity, and the filtering is assumed to commute with time and spatial derivatives. The tilde denotes the Favre (density-weighted) filtering $\widetilde{u}_{i}=\overline{\rho u_{i}} / \bar{\rho}$. The variable $\breve{e}_{t}$ represents the total energy density calculated from the filtered variables, i.e., $\bar{\rho} \breve{e}_{t}=\bar{p} /(\gamma-1)+\bar{\rho} \widetilde{u}_{i} \widetilde{u}_{i} / 2$ for a perfect gas, where $\gamma$ is the specific heat ratio. The resolved viscous stress tensor is defined by $\tilde{\tau}_{i j}=2 \mu\left(\tilde{s}_{i j}-\widetilde{s}_{k k} \delta_{i j} / 3\right)$, where $\tilde{s}_{i j}=\left(\partial_{j} \tilde{u}_{i}\right.$ $\left.+\partial_{i} \tilde{u}_{j}\right) / 2$ and $\mu$ is the molecular dynamic viscosity. The heat flux is given by $\tilde{q}_{j}=-\lambda \partial_{j} \tilde{T}$ where the temperature $\tilde{T}$ is deduced from the filtered density and pressure using the state equation $\bar{p}=\bar{\rho} r \widetilde{T}$, and $\lambda=\mu c_{p} / \sigma$ is the thermal conductivity ( $\sigma$ is the Prandtl number and $c_{p}$ the specific heat at constant pressure). The terms $\sigma_{i}^{\text {sgs }}$ and $\sigma_{e}^{\text {sgs }}$ on the right-hand side of (1) are the so-called subgrid-scale (SGS) terms. A detailed definition of these terms is given in references. ${ }^{32,34}$ The application of a low-pass filtering to the Navier-Stokes equations indeed makes unknown SGS terms appear, which represent the interactions between the resolved scales and the nonresolved scales. An artificial damping is also required as the energy-dissipating scales are lacking. Subgrid models based on eddy viscosity are classically used. However several studies have pointed out some difficulties for high-Reynolds-number flows ${ }^{35}$ or for transitional shear flows ${ }^{27-29,36}$ when such models are implemented.

In the present work, an approach based on a high-order/ selective filtering is developed to take into account the dissipative effects of the subgrid scales, whereas the interac- 
tions between the resolved and the nonresolved scales included in the SGS terms are neglected. The governing equations are solved using low-dispersion and lowdissipation schemes, whereas a selective filtering is applied explicitly to the density, momentum and pressure in order to remove the wave numbers located near the grid cutoff wave number $k_{c}^{g}=\pi / \Delta x$ (where $\Delta x$ is the grid size). Only the fluctuating quantities are involved in the filtering process, which implies, in particular, that it will have no effect in a steady laminar flow. The filtering procedure is described in detail in the next section, but it is interesting to note that it could be integrated into the governing equations. Thus the application of the filter $F$ to the density every iteration corresponds for instance to solving the following mass conservation equation:

$$
\partial_{t} \bar{\rho}+\partial_{j}\left(\bar{\rho} \tilde{u}_{j}\right)=-\left(\sigma_{d} / \Delta t\right) F *(\bar{\rho}-\langle\bar{\rho}\rangle),
$$

where $\sigma_{d}$ is the filtering strength between 0 and $1, \Delta t$ is the time step, and the angular brackets represents a statistical averaging. In the present simulations, the filtering is designed in the Fourier space to eliminate grid-to-grid oscillations without affecting significantly the scales discretized by more than four grid points. The filtering cutoff wave number $k_{c}^{f}=\pi /(2 \Delta x)$ is thus well defined. Further, the amount of dissipation on the larger resolved scales is minimized and the energy is expected to be only diffused when it is transferred to the smaller scales discretized by the mesh grid. This LES approach was recently successfully applied to isotropic turbulence, channel flows, jets and flows around an airfoil, by Visbal et al., ${ }^{37,38}$ Mathew et al., ${ }^{39}$ and the present authors. ${ }^{32,40}$ The explicit selective filtering used here is also very similar ${ }^{41}$ to the relaxation term introduced by Stolz et $a l^{42}$ in their approximate deconvolution model.

\section{B. Numerical procedure}

The numerical algorithm is that used for the simulation of a high-Reynolds-number jet, referred to as LEShre in what follows, and described in detail in an earlier article. ${ }^{32}$ The numerical schemes involved were developed to display optimized properties in the Fourier space so that fluctuations discretized by more than four points per wavelength are neither distorted nor dissipated. ${ }^{30}$ Spatial derivatives are obtained by explicit fourth-order 13-point centered finite differences. The limit of accuracy of this scheme in terms of wave number is $k \Delta x=1.83 \geqslant k_{c}^{f} \Delta x$. The scales that are expected not to be damped by the selective filtering are therefore discretized without significant dispersion. Time integration is performed by a second-order six-stage low-storage RungeKutta algorithm, which is designed to provide very low dispersion and dissipation as shown in Sec. II D.

Selective filtering optimized in the Fourier space to eliminate grid-to-grid oscillations without affecting the scales discretized by more than four grid points are used in order to ensure numerical stability and to take into account the subgrid-scale dissipation as mentioned previously. They are applied explicitly to the density, momentum and pressure every two iterations, sequentially in the $x, y$, and $z$ Cartesian directions and in the diagonals of the $x y, x z$, and $y z$ sections.

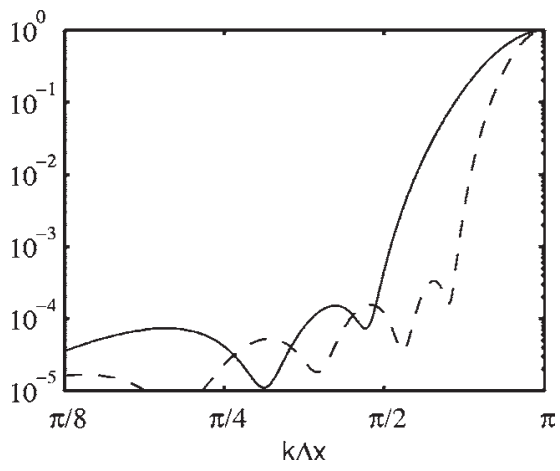

FIG. 1. Damping functions, in logarithmic scales, on a uniform $\Delta x$ grid: of the 13-point selective filter $F^{13}(k \Delta x)(-)$ and of the 21-point filter $F^{21}(k \Delta x)(---)$.

In the Cartesian directions, the filtering is performed using a fourth-order 13-point filter. ${ }^{30}$ Practically, the filtering in the $x$ direction writes, for instance,

$$
U_{i, j, k}^{f}=U_{i, j, k}-\sigma_{d} \sum_{l=-6}^{6} d_{j}^{13}\left(U_{i+l, j, k}-\left\langle U_{i+l, j, k}\right\rangle\right),
$$

where $U=\{\bar{\rho}, \overline{\rho u}, \overline{\rho v}, \overline{\rho w}, \bar{p}\}, U^{f}$ is the filtered variable, angular brackets denote time averages, $d_{j}^{13}$ are the filter coefficients, and the filtering strength is $\sigma_{d}=2 / 3$, arbitrarily. In the diagonals, the filtering is carried out using a fourth-order 21 -point filter, ${ }^{32}$ yielding in the $x y$ direction

$$
U_{i, j, k}^{f}=U_{i, j, k}-\sigma_{d} \sum_{l=-10}^{10} d_{j}^{21}\left(U_{i+l, j+l, k}-\left\langle U_{i+l, j+l, k}\right\rangle\right),
$$

where $d_{j}^{21}$ are the filter coefficients and $\sigma_{d}=2 / 3$ is as previously defined. The diagonal filtering is not applied near the boundaries. Further it is not strictly necessary for stability and it is not used in the latest LES performed in our group. ${ }^{40,43}$ The value of $\sigma_{d}$ and the frequency of filtering are arbitrary, but they are expected to have a negligible influence on the results due to the selectivity of the filters. It was also shown previously that applying the filtering every second or third time step provides very similar features for the highReynolds-number jet LEShre presented in the next sections. ${ }^{29,32}$ In addition, due to the explicit time integration, the time step is only $\Delta t=0.85(D / 30) / c_{0}$, and is quite small with respect to the characteristic time scales of the jet flow. The frequency of application of the selective filtering, every second iteration, therefore appears appropriate for preventing an accumulation of energy at the smaller scales discretized.

To illustrate the properties of the selective filters used in the present LES, the damping functions of these filters are represented in logarithmic scales in Fig. 1, as a function of the wave number $k$ for a uniform grid of mesh size $\Delta x$. The damping function of the 13 -point selective filter ${ }^{30}$ is given by

$$
F^{13}(k \Delta x)=d_{0}^{13}+\sum_{l=1}^{6} 2 d_{j}^{13} \cos (l k \Delta x),
$$

whereas that of the 21-point filter ${ }^{32}$ is provided by 
TABLE I. Mach and Reynolds numbers of the simulated jets, and calculated core lengths $x_{c}$.

\begin{tabular}{cccc}
\hline \hline Jets & $\mathrm{M}$ & $\mathrm{Re}_{D}$ & $x_{c} / r_{0}$ \\
\hline LEShre & 0.9 & $4 \times 10^{5}$ & 10.2 \\
LESre10 & 0.9 & $10^{4}$ & 10.7 \\
LESre5000 & 0.9 & $5 \times 10^{3}$ & 11.3 \\
LESre2500 & 0.9 & $2.5 \times 10^{3}$ & 13.0 \\
LESre1700 & 0.9 & $1.7 \times 10^{3}$ & 15.9 \\
\hline \hline
\end{tabular}

$$
F^{21}(k \Delta x)=d_{0}^{21}+\sum_{l=1}^{10} 2 d_{j}^{21} \cos (l k \Delta x)
$$

The filters are thus shown to dampen the small scales characterized by wave numbers between the filtering cutoff wave number $k_{c}^{f}=\pi /(2 \Delta x)$ and the grid cutoff wave number $k_{c}^{g}=\pi / \Delta x$, while affecting in a negligible manner the large scales such that $k<k_{c}^{f}$, discretized by more than four grid points.

\section{Jet conditions}

Isothermal round jets at Mach number $\mathrm{M}=u_{j} / c_{0}=0.9$ are considered. The initial conditions of the jet flows (shear-layer thickness, inflow forcing) are identical except for the jet diameter $D$, yielding Reynolds numbers $\operatorname{Re}_{D}=u_{j} D / \nu$ ranging from $1.7 \times 10^{3}$ to $4 \times 10^{5}$. In this way, the influence of the Reynolds number on the jet development is investigated in the absence of other initial-condition effects, as recommended by Namer and Ötügen. ${ }^{14}$ The Reynolds numbers of the simulated jets are specified in Table I. The jet LEShre is at the high Reynolds number of $\operatorname{Re}_{D}=4 \times 10^{5}$, whereas the jets LESre10 ${ }^{4}$, LESre5000, LESre2500, and LESre1700 are, respectively, at the low Reynolds numbers of $10^{4}, 5 \times 10^{3}$, $2.5 \times 10^{3}$, and $1.7 \times 10^{3}$.

In the present simulations, the jet inflow conditions are modeled by imposing mean flow profiles while using a random excitation to seed the turbulence. The inflow conditions and forcing are described in detail in the earlier paper where features of the jet LEShre (referred to as LESac in that paper) were first shown to correspond to experimental data obtained for high-Reynolds-number jets. ${ }^{32}$ In practice, the mean axial velocity $u_{0}(r)$ at the jet inflow boundary is given by the following hyperbolic-tangent profile:

$$
\frac{u_{0}(r)}{u_{j}}=\frac{1}{2}+\frac{1}{2} \tanh \left(\frac{r_{0}-r}{2 \delta_{\theta}}\right),
$$

where $u_{j}$ is the inflow centerline velocity, $\delta_{\theta}$ the initial momentum thickness of the annular shear layer, $r_{0}$ the jet radius, $r=\sqrt{y^{2}+z^{2}}$, and the ratio $r_{0} / \delta_{\theta}$ is equal to 20 . Pressure is taken as the ambient pressure, radial and azimuthal velocities are set to zero. The mean density profile $\rho_{0}(r)$ at the inflow is defined by the Crocco-Busemann relation

$$
\frac{\rho_{0}(r)}{\rho_{j}}=\left(1+\frac{\gamma-1}{2} \mathrm{M}^{2} \frac{u_{0}(r)}{u_{j}}\left(1-\frac{u_{0}(r)}{u_{j}}\right)\right)^{-1}
$$

for an isothermal jet ( $\rho_{j}$ is the inflow centerline density). In order to seed the turbulence development, random disturbances are added to the mean velocity profiles in the shear region for $x \simeq r_{0}$, throughout the simulations. The same forcing is applied in the different jets to specify identical inflow conditions. It involves the first 16 azimuthal modes of the jet at the amplitude $\alpha=0.007$, and introduces solenoidal velocity fluctuations with a low magnitude so that it does not generate significant spurious sound waves. It was indeed developed originally with the aim of computing directly the acoustic waves radiated by the jets. ${ }^{33}$ Its influence on jet flow and noise was studied in detail in a previous work, ${ }^{25}$ in which three parameters of the inflow conditions of the LEShre jet are modified: the forcing amplitude, the initial shear-layer thickness and the use of the first modes involved in the forcing. In the same way as in experiments, the flow development was shown to be sensitive to changes in the jet inflow conditions.

\section{Numerical specifications}

A Cartesian grid of $n_{x} \times n_{y} \times n_{z}=255 \times 221 \times 221$ points is used for the simulations. A Cartesian coordinate system is indeed chosen for its accuracy and its simplicity. It allows us in particular to avoid the axis singularity of a cylindrical system. The discretizations in the $y$ and $z$ directions are identical, and are symmetrical about the jet centerline. The transverse mesh spacing is uniform for $y \leqslant 2 r_{0}$ with $\Delta y_{0}=r_{0} / 15$, and then increases at a rate of $2 \%$ to reach $\Delta y=0.4 r_{0}$ well outside the jet flow. The computational domain extends in this way up to $y=16 r_{0}$ from the jet axis. The axial mesh spacing is constant for $0 \leqslant x \leqslant 25 r_{0}$ with $\Delta x=2 \Delta y_{0}$. It is then stretched for $x>25 r_{0}$ in order to form a sponge zone. The time step is $\Delta t=\mathrm{CFL} \times \Delta y_{0} / c_{0}$ with CourantFriedrichs-Lewy number $\mathrm{CFL}=0.85$, and the simulation time is $T=4 \times 10^{4} \Delta t=2270 r_{0} / c_{0}$, which corresponds to $D /\left(T u_{j}\right)=9.9 \times 10^{-4}$ in term of Strouhal number. The computation time is thus long enough to achieve statistical convergence of the results. Finally, nonreflective boundary conditions are used. They are based on the formulation of sound waves in the acoustic far-field, ${ }^{44,45}$ and rely on equations written for the fluctuating quantities alone. Small adjustment terms are therefore introduced near the limits of the computational domain, with the aim of imposing the values of some mean flow quantities at the boundaries. Note however that the mean velocity is not specified outside the jet, in order to allow the incoming of fluid into the computational domain, and consequently to ensure the entrainment of the fluid surrounding the jet into the flow. The detailed implementation of these adjustment terms, as well as additional numerical parameters, can be found in an earlier article. ${ }^{32}$

For the investigation of energy budgets in the jets in Sec. IV A, it is interesting to emphasize the very low dissipation of the present numerical algorithm based on optimized finite-difference and Runge-Kutta schemes. To illustrate this feature, consider a perturbation characterized by the 


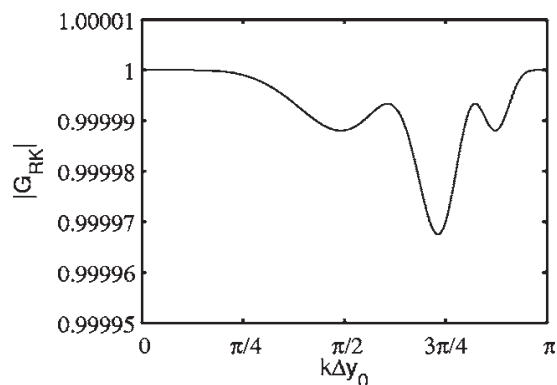

FIG. 2. Amplification rate $\left|G_{\mathrm{RK}}\right|$ per iteration, as a function of the wave number $k \Delta y_{0}$, for waves convected at the velocity $u_{j}$ on the $\Delta y_{0}$ grid in the present simulations. The effects of the selective filtering are not taken into account.

wave number $k$, convected at the velocity $u_{j}$ in the region of the smaller grid spacing $\Delta y_{0}$. The amplification rate per iteration of this wave is given by the amplification factor $\left|G_{\mathrm{RK}}\right|$ of the Runge-Kutta algorithm for the angular frequency $\omega \Delta t=\mathrm{CFL} \times \tilde{k} \Delta y_{0} \times u_{j} / c_{0}$, where $\tilde{k}$ is the effective wave number provided by the finite-difference scheme for the wave number $k$, see Bogey and Bailly ${ }^{30}$ for more details. This amplification rate is presented in Fig. 2. It is very close to 1 for the whole range of wave numbers $0 \leqslant k \Delta y_{0} \leqslant \pi$, and shows in particular that the maximum amount of damping per iteration is of only $3 \times 10^{-5}$. The dissipation due to the discretization schemes, with the exception of the selective filtering, is thus negligible in the present simulations.

\section{JET FLOW FEATURES}

\section{A. Vorticity snapshots}

Snapshots of vorticity are presented in Fig. 3 for the different simulations. The influence of the Reynolds number on the size of the smallest turbulent scales appears clearly. The lower the Reynolds number, the larger the part of the fine scales that are dissipated by the molecular viscosity. This point is remarkably illustrated by the vorticity fields obtained for the jets LEShre and LESre1700: at $\operatorname{Re}_{D}=4 \times 10^{5}$ the turbulent field displays a large range of scales, whereas at $\operatorname{Re}_{D}=1.7 \times 10^{3}$ the most part of the fine scales disappears. This also agrees with experimental observations for circular transitional jets. ${ }^{15,16}$ Further, as the Reynolds number decreases, the generation of vortical structures in the shear layer is found to occur farther downstream. This behavior must be due to the decrease of the growth rates of instability waves by viscosity at low Reynolds numbers. ${ }^{12}$ The length $x_{c}$ of the jet potential core, determined here from the centerline mean velocity $u_{c}$ using $u_{c}\left(x_{c}\right)=0.95 u_{j}$ and reported in Table I, consequently increases as the Reynolds number decreases, in agreement with experimental results obtained for plane ${ }^{14}$ and round ${ }^{15}$ transitional jets. The present core lengths range more precisely from $10.2 r_{0}$ for the LEShre jet up to $15.9 r_{0}$ for the LESre1700. They compare with the experimental data available in the literature which are however quite scattered, the core lengths $x_{c}$ measured by Lau et al. ${ }^{19}$ at $\operatorname{Re}_{D}=10^{6}$, and by Arakeri et al. ${ }^{22}$ at $\operatorname{Re}_{D}=5 \times 10^{5}$ and $\mathrm{Ra}$ man et al. ${ }^{6}$ at $\operatorname{Re}_{D}=4 \times 10^{5}$ in untripped jets varying for instance between $10 r_{0}$ and $14 r_{0}$.

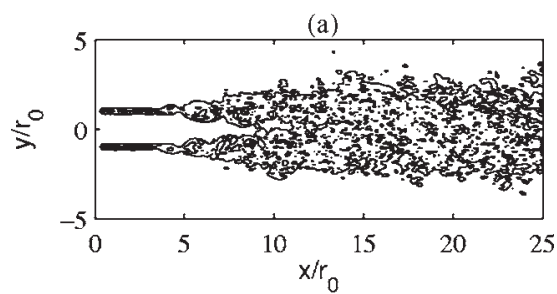

(b)

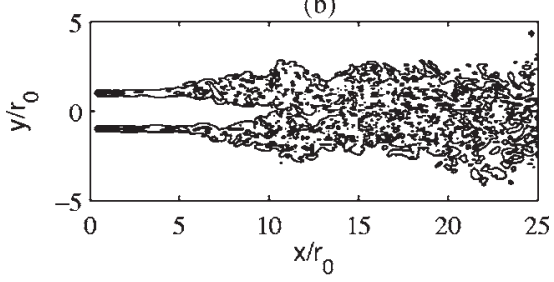

(c)

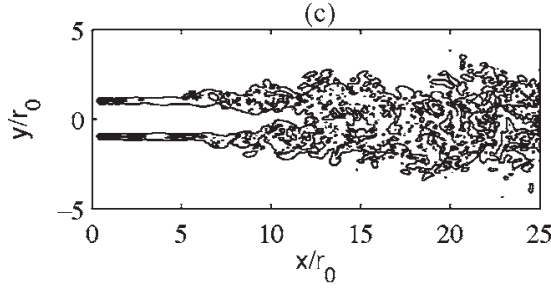

(d)

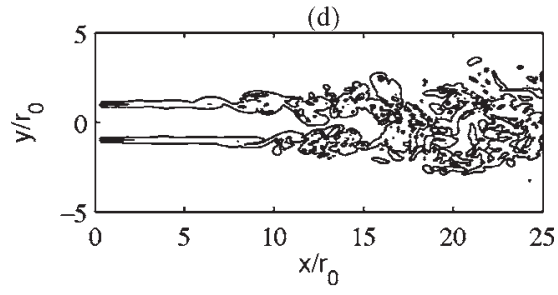

(e)

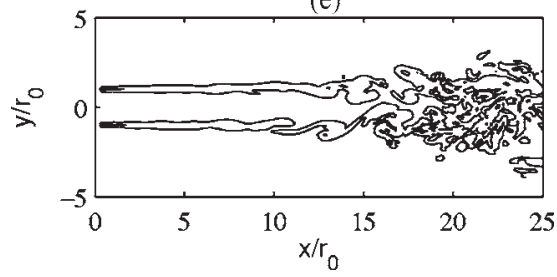

FIG. 3. Snapshots of vorticity in the plane $z=0$. Representation of the two vorticity-norm contours associated with the magnitudes $|\omega| \times r_{0} / u_{j}=[1,4]$ for: (a) LEShre, (b) LESre10 ${ }^{4}$, (c) LESre5000, (d) LESre2500, and (e) LESre1700.

\section{B. Shear-layer development}

In order to investigate the preliminary stage of the shear layer development, the spectra of the fluctuating axial velocity $u^{\prime}$ obtained for $x=3 r_{0}, y=r_{0}$ and $z=0$ are presented in Fig. 4. In all the jets, the velocity spectrum is dominated by a peak at a Strouhal number $\mathrm{St}=f D / u_{j} \simeq 0.6(f$ is the frequency). This peak is connected with the instability waves growing in the initial velocity profiles. Its value compares favorably with the Strouhal number $\mathrm{St}=0.68$ predicted by the linear instability theory ${ }^{12}$ for the most unstable, axisymmetric mode of the inflow shear layer, the discrepancy between the effective and the theoretical Strouhal peaks possibly being due to nonlinear effects and to the differences between the velocity profiles at the inflow and at $x=3 r_{0}$. Moreover, the amplitude of the peaks in the present spectra is found to decrease significantly as the Reynolds number is reduced. This results from the damping influence of viscosity on the 


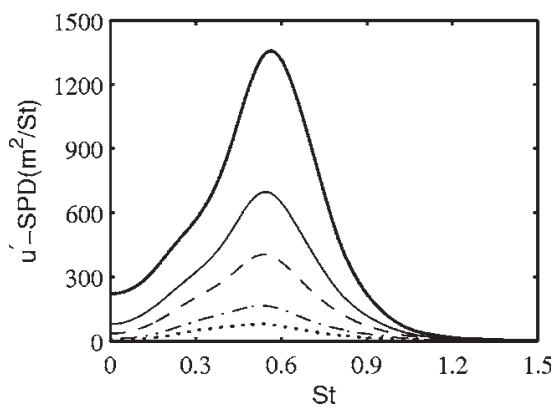

FIG. 4. Spectral power density of $u^{\prime}$ velocity for $x=3 r_{0}, y=r_{0}$, and $z=0$ as a function of Strouhal number $\mathrm{St}=f D / u_{j}$, in linear scales, for: LEShre (-), LESre $10^{4}$ (-), LESre5000 (--), LESre2500 (--), and LESre1700 (*).

growth rate of instability waves, which was for instance described analytically by Morris. ${ }^{46}$ At lower Reynolds numbers, the instability waves thus grow more slowly, which leads to a development of the shear layer that occurs farther downstream, as clearly illustrated in Fig. 5 by the profiles of the shear-layer vorticity thickness and of the level of $u^{\prime}$ velocity for $y=r_{0}$ and $z=0$.

The vorticity thickness $\delta_{\omega}$ of the shear layer, presented in Fig. 5(a) for the different jets, is calculated from the mean axial velocity $\langle u\rangle$ using $\delta_{\omega}=u_{j} / \max (|\partial\langle u\rangle / \partial y|)$, and is related to the momentum thickness $\delta_{\theta}$ for a hyperbolic-tangent profile by $\delta_{\omega}=4 \delta_{\theta}$. For the jet LEShre at high Reynolds number, the vorticity thickness is first fairly constant, and one stage of linear growth is then visibly obtained, in agreement with experimental results of Husain and Hussain ${ }^{47}$ for initially laminar axisymmetric shear layers. The later stage is observed for about $x \geqslant 4 r_{0}$, that is from the location where turbulent structures appear in the shear layer as shown in
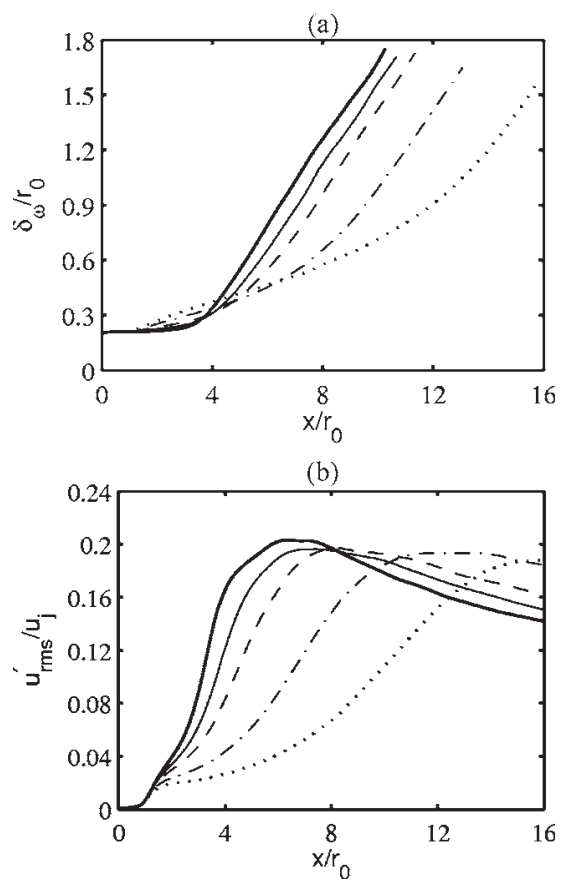

FIG. 5. Axial profiles: (a) vorticity thickness $\delta_{\omega} / r_{0}$ and (b) rms value of $u^{\prime}$ velocity for $y=r_{0}$ and $z=0$, for: LEShre (-), LESre $10^{4}(-)$, LESre5000 (---), LESre2500 (---), and LESre1700 ( ․).
Fig. 3(a). It is characterized by a growth rate $d \delta_{\omega} / d x \simeq 0.22$, which is in the range of the higher rates provided in the literature. ${ }^{48}$ For the jets at lower Reynolds numbers, a stage of linear increase of the vorticity thickness also appears, with a similar growth rate, but it takes place farther downstream, after a transitional zone where the shear layer spreads more slowly. This transitional zone is, for instance, clearly apparent in the jet LEShre1700 for $2 r_{0} \leqslant x \leqslant 12 r_{0}$, which includes a part of the flow where turbulent scales are hardly noticeable, see in Fig. 3(e). The shear layer therefore thickens due to viscosity effects in this case.

The profiles of the $u^{\prime}$-velocity levels along the line $y=r_{0}$ and $z=0$ in Fig. 5(b) finally show the influence of the Reynolds number on the turbulence intensity in the shear layer. The magnitude of the peaks is observed to be slightly smaller at lower Reynolds numbers. The peaks of $u_{\mathrm{rms}}^{\prime} / u_{j}$ are thus for instance of 0.20 in LEShre, and of 0.19 in LESre2500. These values compare well with measurements of Hussain and Zedan ${ }^{49}$ displaying a maximum value of 0.19 in transitional axisymmetric shear layers. The streamwise location of the peaks with respect to the end of the jet core also varies significantly. In the LEShre jet at high Reynolds number, the peak of turbulence intensity in the shear layer occurs at $x \simeq 6 r_{0}$, i.e., four radii upstream from the end of the potential core reported in Table I at $x_{c}=10.2 r_{0}$. At lower Reynolds numbers, the intensity peak is located closer to $x_{c}$. In the LESre2500 jet for example, the peak is only one radius upstream from $x_{c}=13 r_{0}$.

\section{Jet development after the potential core}

In order to investigate the mean flow and turbulence development after the potential core, the streamwise variations of the centerline mean velocity and jet half-width, and of the turbulent axial velocity and turbulence intensity on the jet centerline, are documented in this section.

The profiles of the centerline mean axial velocity $u_{c}$ and of the jet half-width $\delta_{0.5}$ are presented in Fig. 6. For the comparisons, they are shifted axially with respect to the LEShre profiles to match their core lengths $x_{c}$, defined by $u_{c}\left(x_{c}\right)=0.95 u_{j}$ and provided in Table I. As the Reynolds number decreases, the velocity decay and the jet spreading are both found to be more rapid. This influence of the Reynolds number on the velocity decay is supported by the experimental data obtained by Stromberg et al. ${ }^{11}$ and Arakeri et al. ${ }^{22}$ for Mach 0.9, round transitional jets, respectively, at $\mathrm{Re}_{D}=3.6$ $\times 10^{3}$ and $\operatorname{Re}_{D}=5 \times 10^{5}$. In Fig. 6(a), the velocity decay for the jet LESre2500 at $\operatorname{Re}_{D}=2.5 \times 10^{3}$ is indeed similar to that measured for the jet at $\operatorname{Re}_{D}=3.6 \times 10^{3}$, whereas a good agreement is observed between the results for the highReynolds-number jet LEShre and those for the experimental jet at $\operatorname{Re}_{D}=5 \times 10^{5}$. The velocity decay for the jet LEShre is also successfully compared with that provided by Lau et al. ${ }^{19}$ for a Mach 0.9 round jet at $\operatorname{Re}_{D}=10^{6}$. This agreement between results from different jets at high Reynolds numbers seems to indicate that the jet velocity decay is not a function of the Reynolds number above a certain Reynolds number, probably around $\mathrm{Re}_{D} \simeq 10^{5}$. Reynolds-number effects of the same kind as those exhibited by the simulations have been 


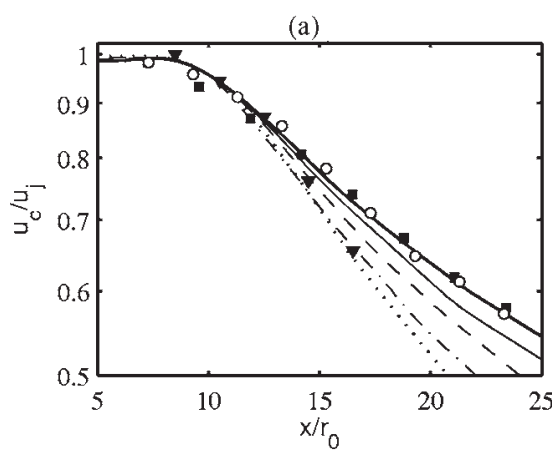

(b)

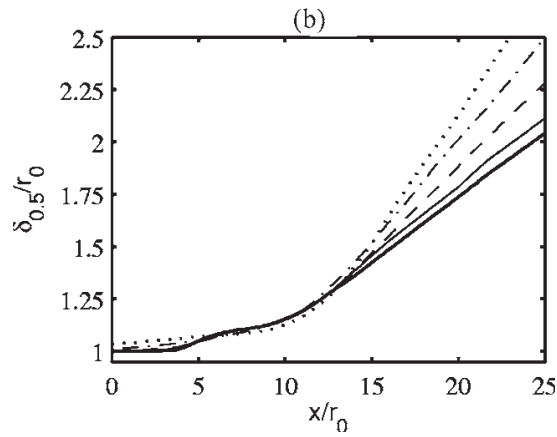

FIG. 6. Axial profiles: (a) mean centerline velocity $u_{c} / u_{j}$ and (b) jet halfwidth $\delta_{0.5} / r_{0}$. Simulations: LEShre (-), LESre $10^{4}(-)$, LESre5000 (---), LESre2500 (-.-), and LESre1700 (..). Measurements: Arakeri et al. (Ref. 22) ( $\mathrm{M}=0.9, \mathrm{Re}_{D}=5 \times 10^{5}$, initially laminar shear layer) (ロ), Lau et al. (Ref. 19) $\left(\mathrm{M}=0.9, \mathrm{Re}_{D}=10^{6}\right)(\bigcirc)$, Stromberg et al. (Ref. 11) $(\mathrm{M}=0.9$, $\left.\operatorname{Re}_{D}=3.6 \times 10^{3}\right)(\mathbf{\nabla})$. For the comparison, the profiles are shifted with respect to the LEShre profiles to yield identical core lengths.

obtained experimentally by Namer and Ötügen ${ }^{14}$ for plane jets with Reynolds numbers between 1000 and 7000, and by Kwon and $\mathrm{Seo}^{15}$ for round jets with $437 \leqslant \mathrm{Re}_{D} \leqslant 5142$. In both cases, lower Reynolds numbers were indeed found to speed up the velocity decay and the jet spreading after the potential core. It must be finally noted that there may be $a$ priori no direct link between the influence of the Reynolds number on the velocity decay and jet spreading after the potential core, and that on the decay constant and spreading rate that are evaluated in the jet self-similar region, far downstream from the jet core, see for instance in the overview of Panchapakesan and Lumley. ${ }^{20}$

The centerline profiles of the turbulent axial velocity $u_{\mathrm{rms}}^{\prime}$ and of the turbulence intensity $u_{\mathrm{rms}}^{\prime} / u_{c}$ are presented in Fig. 7. The profiles for the jet LEShre at $\operatorname{Re}_{D}=4 \times 10^{5}$ agree fairly well with those for the experimental jet of Arakeri et $a .^{22}$ at $\operatorname{Re}_{D}=5 \times 10^{5}$. In Fig. 7(a), as the Reynolds number decreases, the amplitude of the peaks of $u_{\mathrm{rms}}^{\prime}$ increases significantly. Their values are provided in Table II, and range from $0.131 u_{j}$ for the LEShre jet up to $0.178 u_{j}$ for the LESre 1700 jet. This trend is supported by the DNS data obtained by Freund ${ }^{31}$ for a Mach 0.9 jet at $\operatorname{Re}_{D}=3.6 \times 10^{3}$, which compare favorably with the results for the simulated jet at $\operatorname{Re}_{D}=2.5 \times 10^{3}$. It was also displayed experimentally by Namer and Ötügen ${ }^{14}$ for plane jets at low Reynolds numbers.

Regarding the turbulence intensity in Fig. 7(b), its increase along the jet axis is more rapid at lower Reynolds numbers. The values of $u_{\mathrm{rms}}^{\prime} / u_{c}$ just downstream from the jet
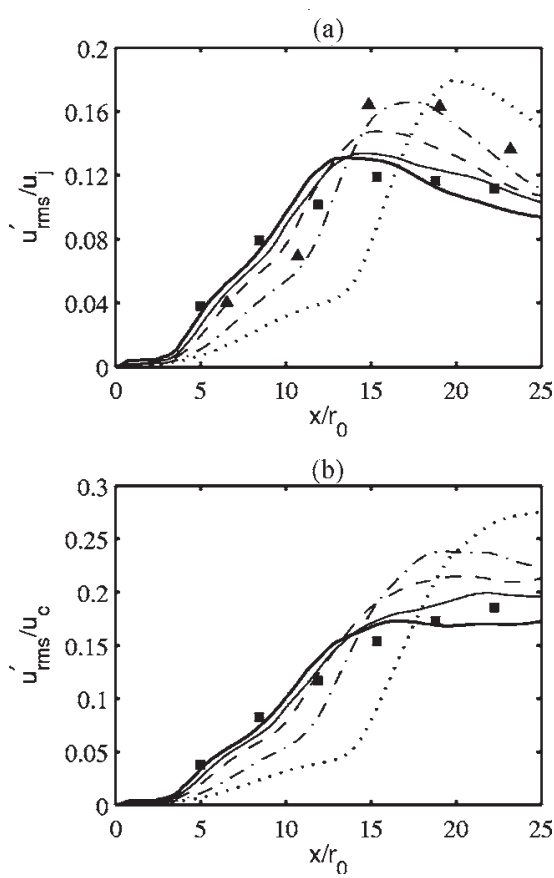

FIG. 7. Centerline profiles: (a) rms fluctuating axial velocity $u_{\mathrm{rms}}^{\prime} / u_{j}$ and (b) turbulence intensity $u_{\mathrm{rms}}^{\prime} / u_{c}$. Simulations: LEShre (-), LESre10 $0^{4}(-)$, LESre5000 (---), LESre2500 (-.-), and LESre1700 ( . -); measurements of Arakeri et al. (Ref. 22) $\left(\mathrm{M}=0.9, \mathrm{Re}_{D}=5 \times 10^{5}\right.$, initially laminar shear-layer) shifted by $-6.5 r_{0}$ for the comparison ( $\left.\mathbf{\square}\right)$; DNS results of Freund (Ref. 31) $\left(\mathrm{M}=0.9, \mathrm{Re}_{D}=3.6 \times 10^{3}\right)$ shifted by $-1.8 r_{0}(\mathbf{\Delta})$.

core thus rise as the Reynolds number decreases. This is clearly illustrated in Table II by the turbulence intensities obtained for the different jets at $x=x_{c}+4 r_{0}$ on the jet axis. At this location, $u_{\mathrm{rms}}^{\prime} / u_{c}$ is for instance equal to 0.162 in the LEShre jet, but to 0.235 for the LESre1700 jet. At the lower Reynolds numbers considered in the present study, the turbulence intensities observed few diameters downstream from the jet core are even close to the value of about 0.25 that is obtained experimentally in the self-similar region of round jets. $^{18,20,21}$ This leads us to expect the jet self-similar region to be reached at a shorter distance at lower Reynolds numbers. This point is also supported by the DNS jet of Freund ${ }^{31}$ at $\operatorname{Re}_{D}=3.6 \times 10^{3}$ where similarity of the turbulent profiles is found for $x \geqslant 25 r_{0}$, whereas at least $x>90 r_{0}$ is required for instance in a jet ${ }^{20,43}$ at $\operatorname{Re}_{D}=1.1 \times 10^{4}$.

TABLE II. Root mean square (rms) values of $\mathrm{u}^{\prime}$ velocity. Left: peak value of $u_{\mathrm{rms}}^{\prime}$ on the jet centerline, right: turbulence intensity for $x=x_{c}+4 r_{0}$ on the jet axis ( $u_{c}$ being the mean centerline velocity).

\begin{tabular}{ccc}
\hline \hline Jets & $\begin{array}{c}\text { Centerline } \\
\text { peak value }\end{array}$ & $\begin{array}{c}x=x_{c}+4 r_{0} \\
y=z=0\end{array}$ \\
\hline LEShre & $0.131 u_{j}$ & $0.162 u_{c}$ \\
LESre10 & $0.134 u_{j}$ & $0.169 u_{c}$ \\
LESre5000 & $0.148 u_{j}$ & $0.189 u_{c}$ \\
LESre2500 & $0.166 u_{j}$ & $0.220 u_{c}$ \\
LESre1700 & $0.178 u_{j}$ & $0.235 u_{c}$ \\
\hline \hline
\end{tabular}




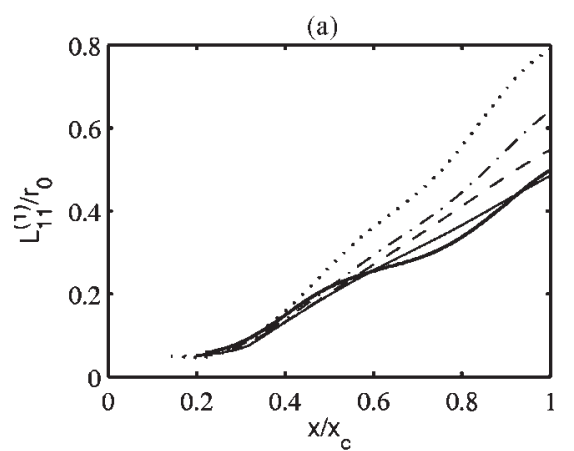

(b)

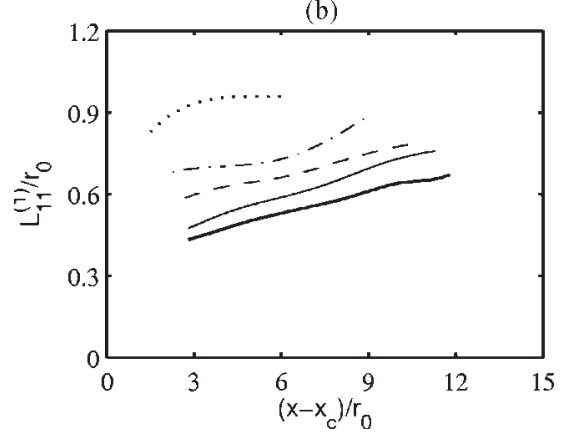

FIG. 8. Axial profiles of the integral length scale $L_{11}^{(1)} / r_{0}$ : (a) in the shear layer for $y=r_{0}$ and $z=0$ as a function of $x / x_{c}$ and (b) on the jet axis as a function of $x-x_{c}$, for: LEShre (-), LESre10 $0^{4}(-)$, LESre5000 (---), LESre2500 (-.-), and LESre1700 ( ․).

\section{Length scales and spectra}

The influence of the Reynolds number on the integral length scales in the jets is now investigated. For this, the longitudinal correlation function of axial fluctuating velocity $R_{11}^{(1)}(r)$ is calculated for points located at $(x, y, z)$ as

$$
R_{11}^{(1)}(r)=\frac{\left\langle u^{\prime}(x+r / 2, y, z) u^{\prime}(x-r / 2, y, z)\right\rangle}{\left\langle u^{\prime 2}(x+r / 2, y, z)\right\rangle^{1 / 2}\left\langle u^{\prime 2}(x-r / 2, y, z)\right\rangle^{1 / 2}}
$$

with $r$ being the separation distance, and the angular brackets denoting time averages. The longitudinal integral scale is then given by $L_{11}^{(1)}=\int_{0}^{\infty} R_{11}^{(1)}(r) d r$.

The normalized integral scales $L_{11}^{(1)} / r_{0}$ obtained for $y=r_{0}$ and $z=0$ in the shear layer are represented in Fig. 8(a), as a function of $x / x_{c}$ in order to take into account the various potential core lengths. In all the jets, they are found to increase with the axial distance. However they display different sizes near the end of the jet core. Larger values of $L_{11}^{(1)} / r_{0}$ are more precisely obtained for $x \simeq x_{c}$ at lower Reynolds

TABLE III. Integral length scales $L_{11}^{(1)}$. Left: for $x=x_{c}$ in the shear layer at the end of the potential core, right: for $x=x_{c}+4 r_{0}$ on the jet axis.

\begin{tabular}{ccc}
\hline \hline & $\begin{array}{c}x=x_{c} \\
y=r_{0} \\
z=0\end{array}$ & $\begin{array}{c}x=x_{c}+4 r_{0} \\
y=0 \\
\text { Jets }\end{array}$ \\
LEShre & $0.50 r_{0}$ & $0.47 r_{0}$ \\
LESre10 & $0.49 r_{0}$ & $0.53 r_{0}$ \\
LESre5000 & $0.55 r_{0}$ & $0.62 r_{0}$ \\
LESre2500 & $0.64 r_{0}$ & $0.70 r_{0}$ \\
LESre1700 & $0.79 r_{0}$ & $0.95 r_{0}$ \\
\hline \hline
\end{tabular}

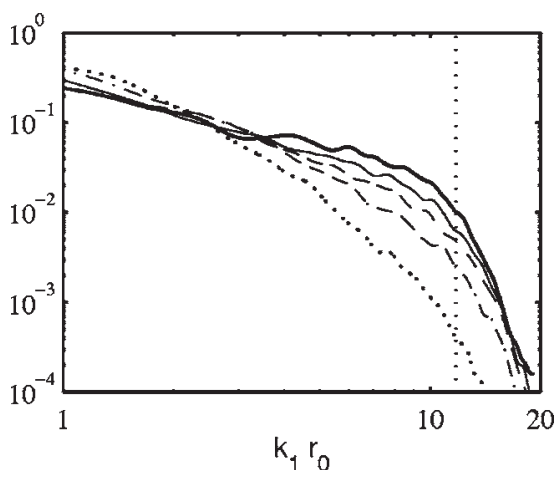

FIG. 9. One-dimensional spectrum $E_{u^{\prime}}^{(1)}\left(k_{1}\right) /\left(r_{0} u_{\mathrm{rms}}^{\prime 2}\right)$ of $u^{\prime}$ velocity for $x=20 r_{0}$ and $y=z=0$, as a function of the normalized axial wave number $k_{1} r_{0}$, for: LEShre (-), LESre10 ${ }^{4}$ (-), LESre5000 (---), LESre2500 (---), and LESre1700 $(\cdots)$. The dotted vertical line represents the filtering cutoff wave number $k_{c}^{f}=\pi /(2 \Delta x)$.

numbers. This Reynolds number effect is illustrated in Table III providing the longitudinal integral scales $L_{11}^{(1)}$ calculated for $x=x_{c}$ in the shear layer. The integral scales at this location are shown to range from $0.50 r_{0}$ for the LEShre jet at high Reynolds number up to $0.79 r_{0}$ for the LESre 1700 jet.

Significant changes due to the Reynolds number also appear in Fig. 8(b) where the centerline longitudinal scales after the jet core are plotted as a function of $x-x_{c}$, that is the axial position with respect to the end of the potential core. As the Reynolds number decreases, the centerline length scales are observed to be of larger size just downstream from the jet core. The values of $L_{11}^{(1)} / r_{0}$ obtained for $x=x_{c}+4 r_{0}$ on the jet axis are for instance reported in Table III. They are found to be noticeably scattered, increasing from $0.47 r_{0}$ for the LEShre jet up to $0.95 r_{0}$ for the LESre 1700 jet. As for the growth rate of the integral scales along the jet axis, it cannot be easily estimated because of the limited computational domain. A previous simulation ${ }^{32}$ using a mesh grid extending up to $x=60 r_{0}$ in the downstream direction nevertheless showed that for the LEShre jet it agrees well with the linear evolution measured by Wygnanski and Fiedler ${ }^{18}$ in a highReynolds-number round jet. In the present simulated jets, the growth of the length scales seems also rather similar, as suggested by the experimental data of Namer and Ötügen ${ }^{14}$ in low-Reynolds-number plane jets for $x \geqslant 60 r_{0}$. Simulations on larger grids are however required to settle this matter.

One-dimensional spectra of $u^{\prime}$ velocity $E_{u^{\prime}}^{(1)}\left(k_{1}\right)$, where $k_{1}$ is the wave number in the axial direction, are calculated. They are obtained from the temporal spectra $E_{u^{\prime}}(f)$, using the Taylor hypothesis of a frozen turbulence yielding $k_{1}=2 \pi f /\langle u\rangle$ and $E_{u^{\prime}}^{(1)}\left(k_{1}\right)=E_{u^{\prime}}(f) \times\langle u\rangle /(2 \pi)$, where $f$ is the frequency and $\langle u\rangle$ is the mean axial velocity. The computations of $E_{u^{\prime}}(f)$ involve the final $3.5 \times 10^{4}$ iterations of the LES, which leads to signal durations corresponding to $1.1 \times 10^{-3}$ in term of minimal Strouhal number. The signals are also divided into 199 overlapping sections. Note that due to the time sampling of the signals the spectra are not calculated up the grid cutoff wave number $k_{c}^{g}=\pi / \Delta x=24 / r_{0}$, but end slightly before, around $k_{1}=20 / r_{0}$.

The spectra $E_{u^{\prime}}^{(1)}\left(k_{1}\right)$ calculated for $x=20 r_{0}$ on the jet cen- 
TABLE IV. Wave numbers associated with the axial integral length scale, the transverse Taylor and Kolmogorov scales $\lambda_{g}$ and $\eta$, for $x=20 r_{0}$ on the jet axis. The filtering and grid cutoff wave numbers are, respectively, $k_{c}^{f}=\pi /(2 \Delta x)=12 / r_{0}$ and $k_{c}^{g}=\pi / \Delta x=24 / r_{0}$.

\begin{tabular}{cccc}
\hline \hline Jets & $1 / L_{11}^{(1)}$ & $1 / \lambda_{g}$ & $1 / \eta$ \\
\hline LEShre & $1.6 / r_{0}$ & $48 / r_{0}$ & $2000 / r_{0}$ \\
LESre10 & $1.4 / r_{0}$ & $7.6 / r_{0}$ & $130 / r_{0}$ \\
LESre5000 & $1.3 / r_{0}$ & $5.5 / r_{0}$ & $84 / r_{0}$ \\
LESre2500 & $1.3 / r_{0}$ & $4.1 / r_{0}$ & $55 / r_{0}$ \\
LESre1700 & $1.0 / r_{0}$ & $3.2 / r_{0}$ & $43 / r_{0}$ \\
\hline \hline
\end{tabular}

terline are presented in Fig. 9 as a function of $k_{1} r_{0}$. They are normalized by $r_{0} u_{\mathrm{rms}}^{\prime 2}$, using the local value of the mean square velocity fluctuations and the jet radius. The spectra are first observed to all collapse in the vicinity of the filtering cutoff wave number $k_{c}^{f}=\pi /(2 \Delta x)=12 / r_{0}$, where $\Delta x$ is the local mesh spacing in the axial direction. They display fairly similar shapes, but narrow significantly as the Reynolds number decreases due to the damping influence of molecular viscosity on the smaller scales discretized. At lower Reynolds numbers, the relative part of turbulent energy contained in the small scales is then reduced, whereas that contained in the large scales is larger. These effects of the Reynolds number are particularly visible for the jet at $\mathrm{Re}_{D}$ $=1700$. They were also reported by Namer and Ötügen ${ }^{14}$ on velocity spectra in plane jets at $x=20 D$. Moreover the classic $k^{-5 / 3}$ law is not clearly found in the present spectra. The wave number associated with the integral length scale is shown below to be about $k_{1}=1.5 / r_{0}$ at the location where the spectra are considered. Thus a zone corresponding to the inertial range of turbulence is likely not to be obtained for the wave numbers that are discretized.

The characteristic wave numbers of the turbulence, associated with the axial integral scale, the transverse Taylor scale $\lambda_{g}$ and the Kolmogorov scale $\eta$, are finally evaluated for $x=20 r_{0}$ on the jet axis, and given in Table IV. The Taylor and Kolmogorov scales are calculated using the relations of isotropic turbulence, $\lambda_{g}=\left(15 L_{11}^{(1)} \nu / u_{\mathrm{rms}}^{\prime}\right)^{1 / 2}$ and $\eta=\left(L_{11}^{(1)}\right)^{1 / 4}\left(\nu / u_{\text {rms }}^{\prime}\right)^{3 / 4}$. In the LEShre jet at high Reynolds number, there is a significant difference between the turbulence scales, with ratios $L_{11}^{(1)} / \lambda_{g}=30$ and $L_{11}^{(1)} / \eta=1250$, and both the Taylor and the Kolmogorov scales are not discretized, the grid cutoff wave number being $k_{c}^{g}=\pi / \Delta x$ $=24 / r_{0}$. At lower Reynolds numbers, these two scales become closer to the integral scale, with ratios decreasing down to $L_{11}^{(1)} / \lambda_{g}=3.2$ and $L_{11}^{(1)} / \eta=43$ in the LESre1700 jet. In the jets at Reynolds numbers $\mathrm{Re}_{D} \leqslant 10^{4}$, the Taylor scale is consequently taken into account by the grid, but is also not affected significantly by the selective filtering as $1 / \lambda_{g}<k_{c}^{f}$. In these jets, the Taylor scale $\lambda_{g}$ is therefore well resolved by the numerical procedure. As for the Kolmogorov scale, it is not discretized even in the jet at $\operatorname{Re}_{D}=1.7 \times 10^{3}$. In that jet, $k_{c}^{f} \eta=0.28$ is in particular obtained, which shows that the simulation LESre1700 is still a LES. One can however note that a sufficiently low Reynolds number would lead to the feature $k_{c}^{f} \eta \geqslant 1.5$ indicative of a DNS. ${ }^{50,51}$

\section{ENERGY DISSIPATION MECHANISMS}

\section{A. Budget of turbulence energy}

The budget of the turbulent kinetic energy $k=\left\langle\bar{\rho} u_{i}^{\prime} / 2\right\rangle$ is calculated in the present jets in order to establish the influence of the Reynolds number on the dissipation mechanisms involved in the LES. The equation expressing this budget is derived from the governing equations (1) given in Sec. II A. It writes

$$
\begin{aligned}
0= & \underbrace{-\frac{\partial}{\partial x_{j}}\left(k\left[u_{j}\right]\right)}_{\text {mean convection }}-\underbrace{\left\langle\bar{\rho} u_{i}^{\prime} u_{j}^{\prime}\right\rangle \frac{\partial\left\lfloor u_{i}\right\rfloor}{\partial x_{j}}}_{\text {production }}-\underbrace{\frac{1}{2} \frac{\partial}{\partial x_{j}}\left\langle\bar{\rho} u_{i}^{\prime 2} u_{j}^{\prime}\right\rangle}_{\text {turbulence diffusion }} \\
& \underbrace{-\frac{\partial}{\partial x_{i}}\left\langle p^{\prime} u_{i}^{\prime}\right\rangle}_{\text {pressure diffusion }}+\left\langle p^{\prime} \frac{\partial u_{i}^{\prime}}{\partial x_{i}}\right\rangle-\left\langle u_{i}^{\prime}\right\rangle \frac{\partial\langle p\rangle}{\partial x_{i}}-\underbrace{\left\langle\tilde{\tau}_{i j} \frac{\partial u_{i}^{\prime}}{\partial x_{j}}\right\rangle}_{\text {viscous dissipation }} \\
& +\frac{\partial}{\partial x_{j}}\left\langle u_{i}^{\prime} \tilde{\tau}_{i j}\right\rangle+D_{\text {filter }},
\end{aligned}
$$

where the prime indicates a fluctuating quantity, the angular brackets denote a statistical average, and $\left[u_{i}\right]=\left\langle\overline{\rho u_{i}}\right\rangle /\langle\bar{\rho}\rangle$. The main terms are those corresponding to mean flow convection, production, viscous dissipation, turbulence diffusion, and pressure diffusion, and the term $D_{\text {filter }}$ represents the filtering dissipation. The budget equation (6) was recently solved by the authors ${ }^{43}$ from the LES fields obtained for a jet at the same Reynolds number as that investigated experimentally by Panchapakesan and Lumley. ${ }^{20}$ Numerical results and measurements in the jet self-similar region were found to be in very good agreement. It was also used ${ }^{29}$ to study the contribution of eddy viscosity in a LES of the jet at $\mathrm{Re}_{D}=4$ $\times 10^{5}$ making use of the dynamic Smagorinsky model.

In the present work, all the terms in the budget equation are calculated explicitly from the LES data, except for the filtering dissipation $D_{\text {filter }}$. In the same way as Dejoan and Leschziner $^{52}$ estimated the dissipation rates in their LES of a plane turbulent wall jet, the filtering contribution is here evaluated as the balance of the other terms. The possibility of doing in this manner is justified by the negligible dissipation of the time-integration algorithm. This matter is addressed in Sec. II D, where the maximum amount of dissipation provided by the discretization schemes, with the exception of the selective filter, for a wave convected at the jet velocity $u_{j}$ at the present CFL number of 0.85 , is shown to be of only $3 \times 10^{-5}$ per iteration.

As an illustration of the LES results, the energy budget obtained across $x=20 r_{0}$ for the high-Reynolds-number jet LEShre is presented in Fig. 10. The viscous dissipation appearing negligible in this jet, the dissipation is only due to the filtering. The present curves cannot be compared directly to the experimental curves of the literature, ${ }^{18,20,21}$ which are provided farther downstream in the self-similar region of the flow. Qualitative similarities are however observed, in particular with the experimental results of Panchapakesan and Lumley. $^{20}$ 


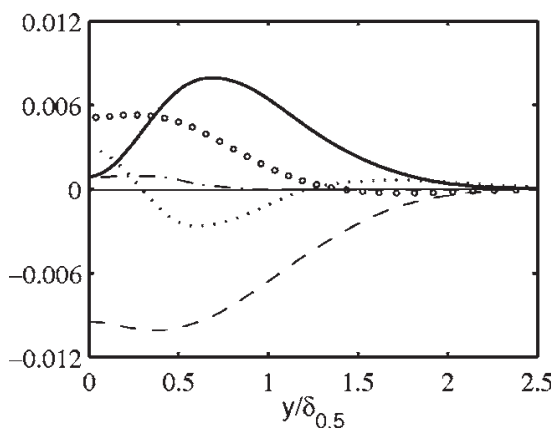

FIG. 10. Kinetic energy budget for $x=20 r_{0}$ from LEShre: mean flow convection $(\bigcirc)$, production $(-)$, viscous dissipation (-), filtering dissipation $(---)$, turbulence diffusion $(\cdots)$, and pressure diffusion (---). The curves are normalized by $\rho_{c} u_{c}^{3} \delta_{0.5}$ ( $\rho_{c}$ being the centerline mean density).

\section{B. Dissipation rates}

The dissipation rates due to the two damping mechanisms involved in the LES are evaluated from the energy budgets across $x=20 r_{0}$ in the different jets, and are presented in Fig. 11. They show that the contributions of molecular viscosity and of the explicit selective filtering to energy dissipation depend closely on the Reynolds number of the flow. As the Reynolds number decreases, the proportion of energy
TABLE V. Contributions to the energy dissipation at $x=20 r_{0}$ on the jet axis.

\begin{tabular}{ccc}
\hline \hline Jets & $\begin{array}{c}\text { Molecular } \\
\text { viscosity }\end{array}$ & $\begin{array}{c}\text { Selective } \\
\text { filtering }\end{array}$ \\
\hline LEShre & 0.01 & 0.99 \\
LESre10 & 0.37 & 0.63 \\
LESre5000 & 0.54 & 0.46 \\
LESre2500 & 0.79 & 0.21 \\
LESre1700 & 0.86 & 0.14 \\
\hline \hline
\end{tabular}

removed by the filtering is found to lessen, whereas that of energy dissipated by viscosity increases. In this way, the filtering is observed to be the dominant process of energy dissipation in Figs. 11(a) and 11(b) for the jets LEShre and LESre $10^{4}$, whereas viscosity predominates in Figs. 11(c)-11(e) for the three jets at low Reynolds numbers $\operatorname{Re}_{D} \leqslant 5 \times 10^{3}$

In the jet at $\operatorname{Re}_{D}=4 \times 10^{5}$ in Fig. 11(a), the contribution of molecular viscosity appears even negligible across $x=20 r_{0}$. Only $1 \%$ of energy dissipation at $x=20 r_{0}$ on the jet axis is for example due to viscosity, as it is reported in Table V. This behavior arises from the features of LES for a high-Reynolds-number flow. At the Reynolds number of the

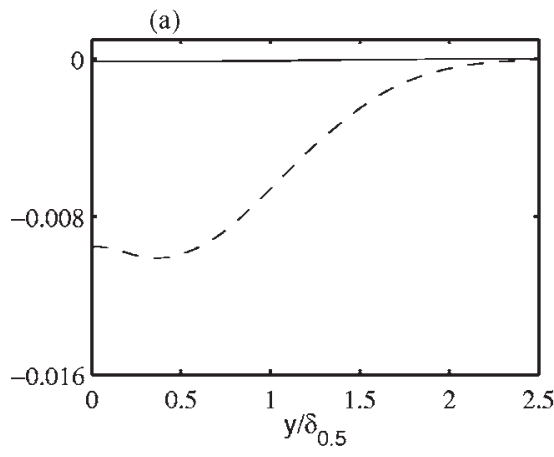

(b)

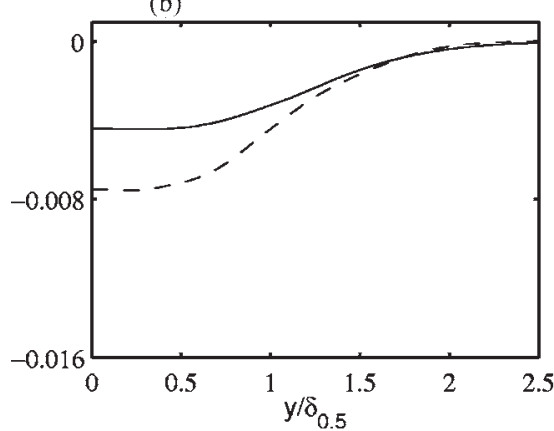

(c)

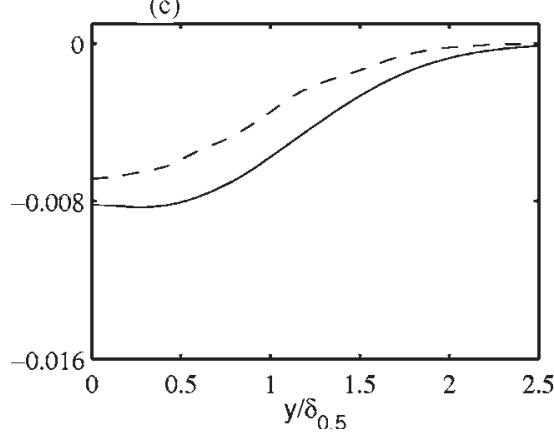

(d)

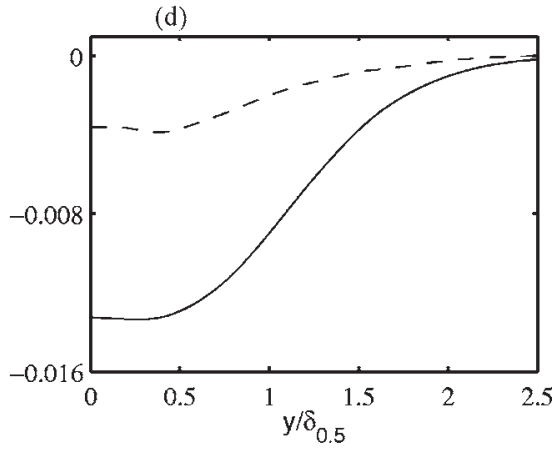

(e)

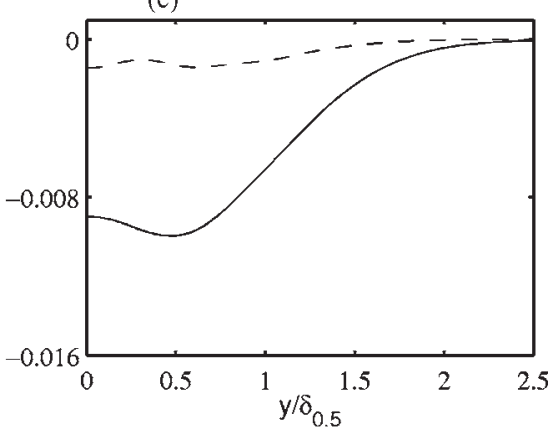

FIG. 11. Dissipation rates for $x=20 r_{0}$, due to: molecular viscosity (-) and filtering (---): (a) LEShre (b) LESre10 ${ }^{4}$ (c) LESre5000, (d) LESre2500, and (e) LESre1700. The curves are normalized by $\rho_{c} u_{c}^{3} \delta_{0.5}$. 
LEShre jet, most of the scales appreciably affected by molecular viscosity are likely to be lacking because they cannot be taken into account by the grid. This point is supported by the sizes of turbulent scales given in Table IV for $x=20 r_{0}$ on the jet centerline. The Taylor scale for which a significant part of the energy should be damped ${ }^{51}$ is for instance shown not to be discretized. Energy dissipation must therefore be yielded almost entirely by the subgrid model, namely by the explicit filtering in the present LES, which leads in Table V to the proportion of $91 \%$ of energy that is filtered out. Note that a simulation of the LEShre jet in which the explicit filtering is applied every three iteration instead of every second iteration was carried out previously. ${ }^{29}$ The dissipation rates were found to be independent of the filtering frequency. This result is certainly due to the high selectivity of the filtering, and suggests that energy is only removed when it is transferred from the large scales to the small scales affected by the filtering.

In the jets at lower Reynolds numbers, the dissipation rates due to molecular viscosity increase, and become progressively predominant. The contribution of viscosity to energy dissipation at $x=20 r_{0}$ on the jet axis thus goes up from 0.37 in the LESre $10^{4}$ jet to 0.86 in the LESre 1700 jet in Table V. This Reynolds number effect results from that documented in Table IV on the turbulent scales. As the Reynolds number decreases, the Taylor and the Kolmogorov scales are indeed larger, and become closer to the integral scale. A greater part of the turbulent scales affected by molecular viscosity are consequently discretized, which implies higher rates of viscous dissipation. In the jet at $\operatorname{Re}_{D}=1.7$ $\times 10^{3}$, the explicit filtering is in particular responsible for only $14 \%$ of energy dissipation at $x=20 r_{0}$ on the jet axis. This nonnegligible contribution shows however that the LESre1700 simulation still requires the use of a model for the effects of the subgrid scales associated with wave numbers between the grid cutoff and the Kolmogorov wave numbers $k_{c}^{g}=\pi / \Delta x$ and $1 / \eta$ given in Table IV, in order to avoid an accumulation of energy at $k=k_{c}^{g}$. DNS could be obtained at lower Reynolds numbers provided that all turbulence scales be accurately resolved by the numerical procedure, i.e., that their corresponding wave numbers are so that $k<k_{c}^{f}$. For a DNS level of resolution, the contribution of the filtering to energy dissipation is expected to be very small. When the discretization schemes provide negligible dissipation, as in the present simulations, see in Fig. 2, the greater part of the energy dissipation will then be due to molecular viscosity. However it might not be the case using a more dissipative numerical approach.

\section{Analysis in the wave number space}

The features of the dissipation mechanisms in the wave number space are finally examined by characterizing the turbulent scales that are affected by molecular viscosity and by the explicit filtering for $x=20 r_{0}$ on the jet axis. With this aim in view, the following quantities are calculated in the different simulations: $\nu k_{1}^{2} E_{u^{\prime}}^{(1)}\left(k_{1}\right)$ for molecular viscosity, and $D_{f}\left(k_{1} \Delta x\right) E_{u^{\prime}}^{(1)}\left(k_{1}\right)$ for the filtering, where $k_{1}$ is the axial wave number, $E_{u^{\prime}}^{(1)}\left(k_{1}\right)$ is the one-dimensional spectrum of $u^{\prime}$ ve-

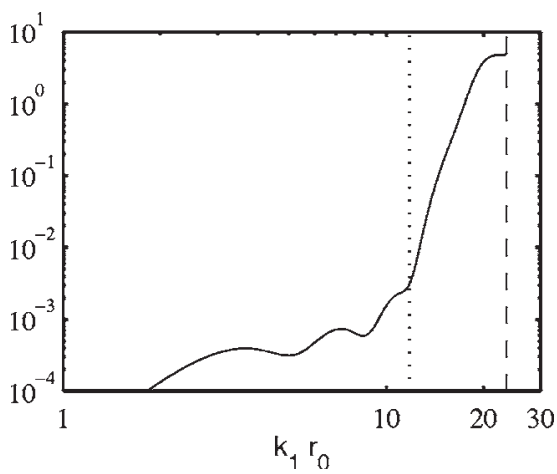

FIG. 12. Transfer function $D_{f}\left(k_{1} \Delta x\right) \times\left(r_{0} / u_{j}\right)$ of the filtering procedure in the axial direction, in logarithmic scales, as a function of $k_{1} r_{0}$; filtering cutoff wave number $k_{c}^{f}=\pi /(2 \Delta x)(\cdots)$ and grid cutoff wave number $k_{c}^{g}=\pi / \Delta x(---)$.

locity for $x=20 r_{0}$ and $y=z=0$ presented in Fig. 9, and $D_{f}\left(k_{1} \Delta x\right)$ is the transfer function in the axial direction of the explicit filtering procedure.

To evaluate the transfer function $D_{f}\left(k_{1} \Delta x\right)$, consider for instance the 13-point filtering carried out in the axial direction on the uniform grid of mesh size $\Delta x$, which is given by expression (2) in Sec. II B. Its application every second time step is equivalent to a second-order explicit integration over the time period $2 \Delta t$ of the following term

$$
D_{f}^{13}\left(U_{i, j, k}^{\prime}\right)=\frac{\sigma_{d}}{2 \Delta t} \sum_{l=-6}^{6} d_{j}^{13} U_{i+l, j, k}^{\prime}
$$

in the left-hand side of the governing equations (1). The transfer function of this filtering operation is then obtained using spatial Fourier transform in the axial direction, yielding

$$
D_{f}^{13}\left(k_{1} \Delta x\right)=\sigma_{d} F^{13}\left(k_{1} \Delta x\right) /(2 \Delta t),
$$

where $F^{13}\left(k_{1} \Delta x\right)$ is the damping function of the 13-point filter on the $\Delta x$ grid, precedently given by expression (4) and presented in Fig. 1.

In the present simulations, the flow variables are filtered every second iteration sequentially in the $x, y$, and $z$ Cartesian directions using the 13-point filter and in the diagonals of the $x y, x z$ and $y z$ sections using a 21-point filter, as reported in Sec. II B. The transfer function in the axial direction of this procedure involving multiple filterings is thus

$$
\begin{aligned}
D_{f}\left(k_{1} \Delta x\right)= & {\left[1-\left(1-\sigma_{d} F^{13}\left(k_{1} \Delta x\right)\right)\right.} \\
& \left.\times\left(1-\sigma_{d} F^{21}\left(k_{1} \Delta x\right)\right)^{4}\right] /(2 \Delta t),
\end{aligned}
$$

where $F^{21}\left(k_{1} \Delta x\right)$ is the damping function of the 21-point filter on the $\Delta x$ grid, see expression (5) and Fig. 1. The transfer function (7) is represented in Fig. 12 as a function of $k_{1} r_{0}$ for the different simulations. The curves obtained collapse onto one curve because of the normalization using $u_{j}$ and $r_{0}$. As we could expect from the properties of the filters, the effects of the filtering procedure are shown to be maximum at the grid cutoff wave number $k_{c}^{g}$, and to fall off sharply for lower wave numbers. As a result, the amount of dissipation provided by the filtering on the scales so that $k<k_{c}^{f}$, discretized by more than four grid points, is at least of 

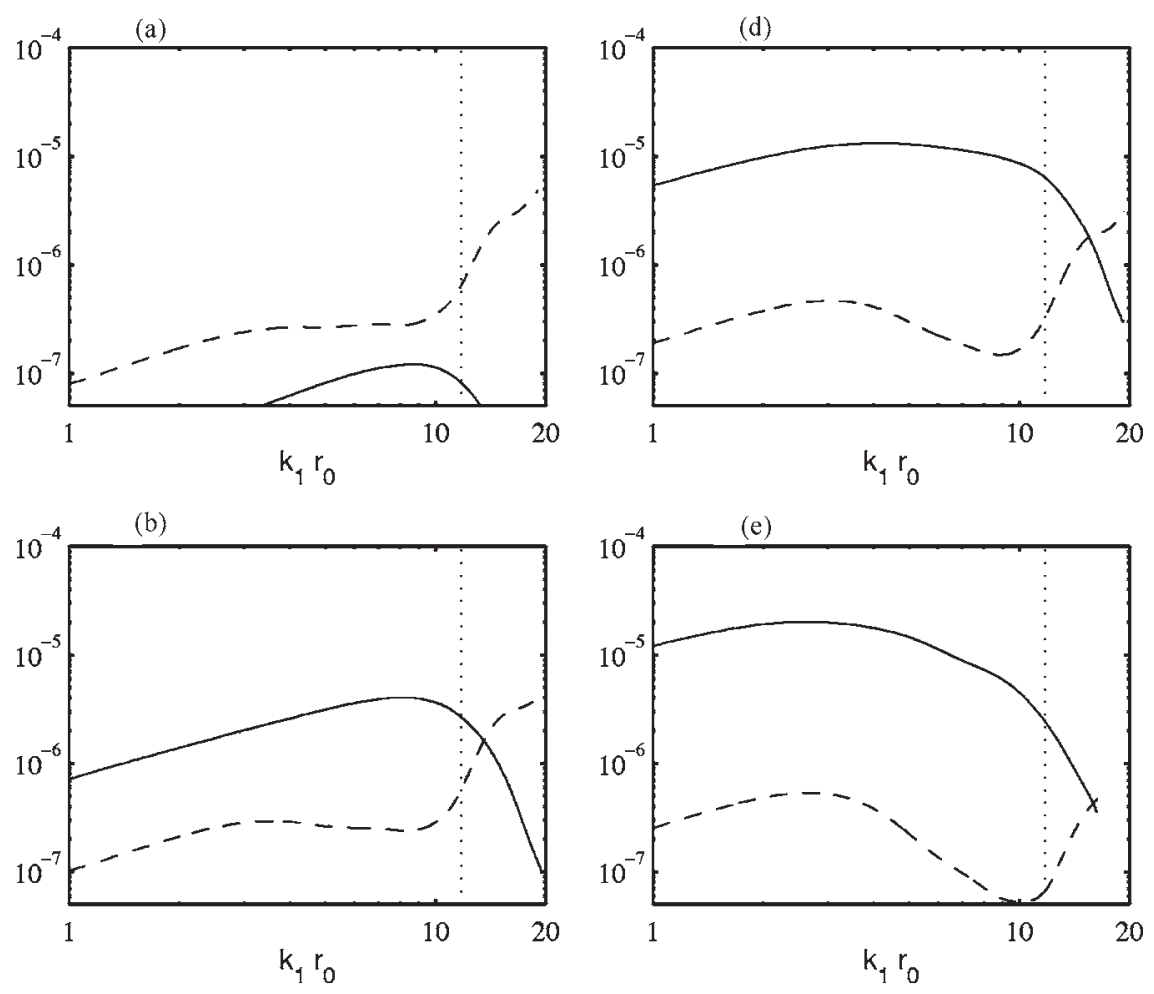

FIG. 13. Features of the dissipation mechanisms for $x=20 r_{0}$ and $y=z=0$, as a function of the axial wave number $k_{1} r_{0}:$ molecular viscosity (-) and filtering (---): (a) LEShre, (b) LESre $10^{4}$, (c) LESre5000, (d) LESre2500, and (e) LESre1700. The dotted vertical line represents the filtering cutoff wave number $k_{c}^{f}=\pi /(2 \Delta x)$. The curves are normalized by $u_{j}^{3}$.

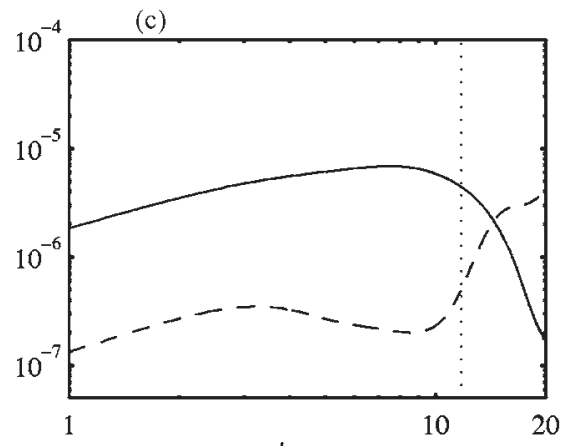

four order of magnitudes lower than that on the grid-to-grid oscillations at $k=k_{c}^{g}$.

The features of the dissipation mechanisms for $x=20 r_{0}$ on the jet axis are now shown in Fig. 13 as a function of $k_{1} r_{0}$. The curves represent the quantities defined earlier, $\nu k_{1}^{2} E_{u^{\prime}}^{(1)}$ $\times\left(k_{1}\right)$ for molecular viscosity and $D_{f}\left(k_{1} \Delta x\right) E_{u^{\prime}}^{(1)}\left(k_{1}\right)$ for the filtering, normalized by $u_{j}^{3}$. The filtering cutoff wave number $k_{c}^{f}=\pi /(2 \Delta x)$ is also indicated. It must be moreover noted that the dissipation curves are not obtained up to the grid cutoff wave number $k_{c}^{g}=\pi / \Delta x=24 / r_{0}$, but up to the maximum wave number $k_{1} \simeq 20 / r_{0}$ for which the velocity spectra could have been calculated, see Fig. 9 in Sec. III D. Selective filtering and molecular viscosity are observed to dissipate energy through different turbulence scales: filtering through the smallest scales discretized, which are located beyond the filtering cutoff wave number $k_{c}^{f}$, and molecular viscosity through a wide range of scales including the larger ones. This difference is for instance clearly illustrated in Fig. 13(b), for the LESre $10^{4}$ jet in which the contributions of the two damping mechanisms are similar as displayed in Table V.

For the LEShre jet in which viscous dissipation is negligible because of the high Reynolds number, energy is found in Fig. 13(a) to be mainly damped by the explicit filtering through the high wave numbers so that $k_{1}>k_{c}^{f}$. The amount of energy dissipated through the large scales associated with wave numbers $k_{1}<k_{c}^{f}$ is at least one order of magnitude lower than that observed for the smaller scales. In the present LES, the effects of the explicit filtering on the large scales are however stronger than those of molecular viscosity. This shows that for flows at high Reynolds numbers, the effects of molecular viscosity being insignificant on the large turbulent structures, it is difficult to avoid that numerical dissipation does not exceed physical dissipation due to viscosity on these structures, even when very low dissipation space and time discretization schemes are used. Ideally this requirement should be met, but this would require numerical schemes even less dissipative than the optimized schemes presently used.

In Figs. 13(a)-13(d), one can finally study the changes in the shape of the dissipation curve associated with molecular viscosity as the Reynolds number varies. For the four jets at low Reynolds numbers $\operatorname{Re}_{D} \leqslant 10^{4}$, the viscous dissipation appears to affect significantly a wide range of scales including the larger ones. This is the case for instance for the scale 
associated with the wave number $k_{1} r_{0}=1$, which is discretized by 47 grid points. The peaks of viscous dissipation are obtained for wave numbers lower than the filtering cutoff wave number $k_{c}^{f}$. These peak wave numbers are in good agreement ${ }^{51}$ with the wave numbers $1 / \lambda_{g}$ associated with the transverse Taylor scales, which are estimated in Table IV using the relations of isotropic turbulence, and range from $1 / \lambda_{g}=7.6 r_{0}$ for the LESre 10 jet down to $1 / \lambda_{g}=3.2 r_{0}$ for the LESre1700 jet. Following the variations of the Taylor scale, the dissipation peak is thus shifted toward lower wave numbers with decreasing Reynolds number.

\section{CONCLUSION}

Large eddy simulations of isothermal transitional round jets at Mach number $\mathrm{M}=0.9$ have been carried out using a LES methodology combining low dissipation and low dispersion discretization schemes with explicit selective filtering of the flow variables. The inflow conditions of the jets are identical except for the Reynolds numbers $\operatorname{Re}_{D}$ ranging from $1.7 \times 10^{3}$ to $4 \times 10^{5}$, which has allowed us to investigate the influence of the Reynolds number on the jet characteristics in the absence of other initial-condition effects. The changes in the features of the dissipation mechanisms involved in the LES, namely molecular viscosity and explicit filtering, with the Reynolds number have been also displayed.

The development of the jet mean flow and turbulence has been shown to be significantly modified as the Reynolds number varies, both in the initial shear layer and in the transitional region located just downstream from the potential core. The Reynolds-number effects observed in the simulated jets are moreover consistent with measurements of the literature, and in particular with the trends exhibited experimentally for low-Reynolds-number jets. The jets develop for instance more rapidly after the potential core, with higher turbulence intensities and larger integral scales, as the Reynolds number decreases. As for the dissipation mechanisms, their contributions to energy dissipation in the LES have been shown to depend closely on the Reynolds number. At high Reynolds number, most of the dissipation scales are not discretized, and energy is mainly damped by the explicit filtering. However the effects of molecular viscosity increase appreciably at lower Reynolds numbers as more dissipation scales are resolved. The scales that are affected by the two damping mechanisms are also characterized. The selective filtering removes the small scales discretized by very few grid points, typically between 2 and 4, whereas molecular viscosity damps a large range of turbulent scales, with a dissipation peak around the transverse Taylor scale.

The present simulations have provided some features regarding the development of turbulent jets that cannot be easily obtained experimentally, as pointed out for instance by Namer and Ötügen. ${ }^{14}$ This is however feasible only because the LES methodology, which has been carefully described by calculating in particular dissipation rates from kinetic energy budgets, appears appropriate for studying Reynolds-number effects. With the increase of computational resources, future works will deal with the self-similar region of the jet, ${ }^{43}$ which is located far downstream from the inflow. The influence of the Reynolds number on the flow properties in this region, including velocity decay and spreading rates, turbulence intensities and energy budgets, could be considered.

\section{ACKNOWLEDGMENTS}

The authors gratefully acknowledge the Institut du Développement et des Ressources en Informatique Scientifique (IDRIS-CNRS) for providing computing time and for its technical assistance.

${ }^{1}$ W. G. Hill Jr., R. C. Jenkins, and B. L. Gilbert, "Effects of the initial boundary-layer state on turbulent jet mixing," AIAA J. 14, 1513 (1976).

${ }^{2}$ J. C. Lau, "Effects of exit Mach number and temperature on mean-flow and turbulence characteristics in round jets," J. Fluid Mech. 105, 193 (1981).

${ }^{3}$ K. B. M. Q. Zaman, "Asymptotic spreading rate of initially compressible jets-experiment and analysis," Phys. Fluids 10, 2652 (1998).

${ }^{4}$ T. G. Malmström, A. T. Kirkpatrick, B. Christensen, and K. D. Knappmiller, "Centreline velocity decay measurements in low-velocity axisymmetric jets," J. Fluid Mech. 346, 363 (1997).

${ }^{5}$ E. Ferdman, M. V. Ötügen, and S. Kim, "Effect of initial velocity profile on the development of round jet," J. Power Sources 16, 676 (2000).

${ }^{6}$ G. Raman, E. J. Rice, and E. Reshotko, "Mode spectra of natural disturbances in a circular jet and the effect of acoustic forcing," Exp. Fluids 17, 415 (1994).

${ }^{7}$ R. A. Antonia and Q. Zhao, "Effect of initial conditions on a circular jet," Exp. Fluids 31, 319 (2001).

${ }^{8}$ G. Xu and R. A. Antonia, "Effects of different initial conditions on a turbulent free jet," Exp. Fluids 33, 677 (2002).

${ }^{9}$ K. B. M. Q. Zaman, "Effect of initial condition on subsonic jet noise," AIAA J. 23, 1370 (1985).

${ }^{10}$ D. K. McLaughlin, G. L. Morrison and T. R. Troutt, "Reynolds number dependence in supersonic jet noise," AIAA J. 15, 526 (1977).

${ }^{11}$ J. L. Stromberg, D. K. McLaughlin, and T. R. Troutt, "Flow field and acoustic properties of a Mach number 0.9 jet at a low Reynolds number," J. Sound Vib. 72, 159 (1980).

${ }^{12}$ A. Michalke, "Survey on jet instability theory," Progress in Aerospace Sciences 21, 159 (1984).

${ }^{13}$ G. P. Lemieux and P. H. Oosthuizen, "Experimental study of the behavior of plane turbulent jets at low Reynolds numbers," AIAA J. 23, 1845 (1985).

${ }^{14}$ I. Namer and M. V. Ötügen, "Velocity measurements in a plane turbulent air jet at moderate Reynolds numbers," Exp. Fluids 6, 387 (1988).

${ }^{15}$ S. J. Kwon and I. W. Seo, "Reynolds number effects on the behavior of a non-buoyant round jet," Exp. Fluids 38, 801 (2005).

${ }^{16}$ T. H. Weisgraber and D. Liepmann, "Turbulent structure during transition to self-similarity in a round jet," Exp. Fluids 14, 210 (1998).

${ }^{17}$ K. B. M. Q. Zaman, "Far-field noise of a subsonic jet under controlled excitation,” J. Fluid Mech. 152, 83 (1985).

${ }^{18}$ I. Wygnanski and H. Fiedler, "Some measurements in the self-preserving jet,” J. Fluid Mech. 38, 577 (1969).

${ }^{19}$ J. C. Lau, P. J. Morris, and M. J. Fisher, "Measurements in subsonic and supersonic free jets using a laser velocimeter," J. Fluid Mech. 93, 1 (1979).

${ }^{20}$ N. R. Panchapakesan and J. L. Lumley, "Turbulence measurements in axisymmetric jets of air and helium. Part I. Air jet," J. Fluid Mech. 246, 197 (1993).

${ }^{21}$ H. J. Hussein, S. P. Capp, and W. K. George, "Velocity measurements in a high-Reynolds-number, momentum-conserving, axisymmetric, turbulent jet," J. Fluid Mech. 258, 31 (1994).

${ }^{22}$ V. H. Arakeri, A. Krothapalli, V. Siddavaram, M. B. Alkislar, and L. Lourenco, "On the use of microjets to suppress turbulence in a Mach 0.9 axisymmetric jet," J. Fluid Mech. 490, 75 (2003).

${ }^{23}$ B. J. Boersma, G. Brethouwer, and F. T. M. Nieuwstadt, "A numerical simulation on the effect of the inflow conditions on the self-similar region of a round jet," Phys. Fluids 10, 899 (1998). 
${ }^{24}$ S. A. Stanley and S. Sarkar, "Influence of nozzle conditions and discrete forcing on turbulent planar jets," AIAA J. 38, 1615 (2000).

${ }^{25}$ C. Bogey and C. Bailly, "Effects of inflow conditions and forcing on subsonic jet flows and noise," AIAA J. 43, 1000 (2005).

${ }^{26}$ M. Klein, A. Sadiki, and J. Janicka, "Investigation of the influence of the Reynolds number on a plane jet using direct numerical simulation," Int. J. Heat Fluid Flow 24, 785 (2003).

${ }^{27}$ J. A. Domaradzki and P. P. Yee, "The subgrid-scale estimation model for high Reynolds number turbulence," Phys. Fluids 12, 193 (2000).

${ }^{28} \mathrm{C}$. Bogey and C. Bailly, "Decrease of the effective Reynolds number with eddy-viscosity subgrid-scale modeling," AIAA J. 43, 437 (2005).

${ }^{29} \mathrm{C}$. Bogey and C. Bailly, "Large eddy simulations of round free jets using explicit filtering with/without dynamic Smagorinsky model,” Int. J. Heat and Fluid Flow (to be published); see also in Proceedings of Turbulence and Shear Flow Phenomena-4, 2005, Vol. 2, p. 817.

${ }^{30} \mathrm{C}$. Bogey and C. Bailly, "A family of low dispersive and low dissipative explicit schemes for flow and noise computations," J. Comput. Phys. 194, 194 (2004).

${ }^{31}$ J. B. Freund, "Noise sources in a low-Reynolds-number turbulent jet at Mach 0.9," J. Fluid Mech. 438, 277 (2001).

${ }^{32}$ C. Bogey and C. Bailly, "Computation of a high Reynolds number jet and its radiated noise using LES based on explicit filtering," Computers and Fluids (to be published).

${ }^{33} \mathrm{C}$. Bogey and C. Bailly, "Investigation of downstream and sideline subsonic jet noise using Large Eddy Simulation," Theor. Comput. Fluid Dyn. 20, 23 (2006).

${ }^{34}$ B. Vreman, B. Geurts, and H. Kuerten, "Subgrid-modelling in LES of compressible flow," Appl. Sci. Res. 54, 191 (1995).

${ }^{35}$ D. H. Porter, P. R. Woodwrad, and A. Pouquet, "Inertial range structures in decaying compressible turbulent flows," Phys. Fluids 10, 237 (1998).

${ }^{36}$ P. Schlatter, S. Stolz, and L. Kleiser, "LES of transitional flows using the approximate deconvolution model," Int. J. Heat Fluid Flow 25, 549 (2004).

${ }^{37}$ M. R. Visbal and D. P. Rizzetta, "Large-eddy simulation on curvilinear grids using compact differencing and filtering schemes," J. Fluids Eng. 124, 836 (2002).
${ }^{38}$ D. P. Rizzetta, M. R. Visbal, and G. A. Blaisdell, "A time-implicit highorder compact differencing and filtering scheme for large-eddy simulation," Int. J. Numer. Methods Fluids 42, 665 (2003).

${ }^{39}$ J. Mathew, R. Lechner, H. Foysi, J. Sesterhenn, and R. Friedrich, "An explicit filtering method for large eddy simulation of compressible flows," Phys. Fluids 15, 2279 (2003).

${ }^{40}$ O. Marsden, C. Bogey, and C. Bailly, "Noise radiated by a high-Reynoldsnumber 3-D airfoil," Proceedings of 11th AIAA/CEAS Aeroacoustics Conference, Monterey, CA, 23-25 May 2005, AIAA Paper 2005-2817.

${ }^{41}$ C. Bailly and C. Bogey, "Current understanding of jet noise-generation mechanisms from compressible large-eddy-simulations," Proceedings of Direct and Large-Eddy Simulation-6, Poitiers, France, 12-14 September 2005.

${ }^{42}$ S. Stolz, N. A. Adams, and L. Kleiser, "An approximate deconvolution model for large-eddy simulation with application to incompressible wallbounded flows," Phys. Fluids 13, 997 (2001).

${ }^{43} \mathrm{C}$. Bogey and C. Bailly, "Computation of the self-similarity region of a turbulent round jet using large eddy simulation," Proceedings of Direct and Large-Eddy Simulation-6, Poitiers, France, 12-14 September 2005.

${ }^{44}$ C. Bogey, C. Bailly, and D. Juvé, "Noise investigation of a high subsonic, moderate Reynolds number jet using a compressible LES," Theor. Comput. Fluid Dyn. 16, 273 (2003).

${ }^{45}$ C. Bogey and C. Bailly, "Three-dimensional non reflective boundary conditions for acoustic simulations: far-field formulation and validation test cases," Acta Acust. 88, 463 (2002).

${ }^{46}$ P. J. Morris, "The spatial viscous instability of axisymmetric jets," J. Fluid Mech. 77, 511 (1976).

${ }^{47}$ Z. D. Husain and A. K. M. F. Hussain, "Axisymmetric mixing layer: influence of the initial and boundary conditions," AIAA J. 17, 48 (1979).

${ }^{48} \mathrm{E}$. Gutmark and C.-M. Ho, "Preferred modes and the spreading rates of jet," Phys. Fluids 26, 2932 (1983).

${ }^{49}$ A. K. M. F. Hussain and M. F. Zedan, "Effects of the initial condition on the axisymmetric free shear layer: Effects of the initial momentum thickness," Phys. Fluids 21, 1100 (1978).

${ }^{50}$ S. B. Pope, Turbulent Flows (Cambridge University Press, Cambridge, UK, 2000).

${ }^{51}$ C. Bailly and G. Comte-Bellot, Turbulence (CNRS Editions, Paris, 2003).

${ }^{52}$ A. Dejoan and M. A. Leschziner, "Large eddy simulation of a plane turbulent wall jet," Phys. Fluids 17, 025102 (2005). 